\title{
A METHOD FOR THE REDUCTION OF SHIP-DETECTION FALSE ALARMS DUE TO SAR AZIMUTH AMBIGUITY
}

\author{
Corrado Avolio ${ }^{2}$, Mario Costantini ${ }^{2}$, Gerardo Di Martino ${ }^{1}$, Antonio Iodice ${ }^{1}$, \\ Flavia Macina ${ }^{2}$, Giuseppe Ruello ${ }^{1}$, Daniele Riccio ${ }^{1}$, Massimo Zavagli ${ }^{2}$ \\ ${ }^{1}$ Università di Napoli Federico II - DIETI, Naples, Italy; \\ ${ }^{2}$ e-Geos, Rome, Italy.
}

\begin{abstract}
Due to the finite pulse repetition frequency and non-ideal antenna pattern, the presence of "ghosts" on SAR images of maritime scenes is frequently observed. This phenomenon can lead to an increase in false alarm rate in ship-detection applications. In this paper we propose to use the recently developed "asymmetric mapping and selective filtering" (AM\&SF) method for the filtering of azimuth ambiguities on stripmap SAR images as a preliminary step of an adaptive-threshold cell-averaging constant-false-alarm-rate ship-detection algorithm. We show that use of this preliminary filtering step allows us to significantly improve the performance of the ship detection by reducing the false alarm rate, without reducing the detection rate. The proposed framework is positively applied to a couple of Cosmo/SkyMed SAR images.
\end{abstract}

Index Terms - SAR, ship detection, azimuth ambiguity

\section{INTRODUCTION}

Use of a finite pulse repetition frequency (PRF), and the fact that azimuth antenna pattern (AAP), in practice, cannot be designed to be abruptly limited to the specified azimuth illumination beamwidth, give rise to the well-known SAR azimuth ambiguity problem. Due to this effect, on SAR images replicas (or "ghosts") of brilliant points or areas appear, that are shifted both in azimuth and (although in a smaller extent) in range. These replicas are strongly attenuated, due to the azimuth antenna beam pattern and because they are not well focused. However, if these "ghosts" are placed in a low-scattering area, such as the sea surface, they emerge with respect to the background and can be erroneously interpreted as actual targets. Accordingly, for ship-detection applications, ghosts of strong scatterers placed on dry land (e.g., buildings) or also on the sea (e.g., other ships) may cause false alarms [1].

In the literature, several ways are known for the suppression of azimuth ambiguities [1]-[8]. Among them, some are suitable only for pointlike scatterers [1]-[4] (unless further post-processing is applied [4]) and they can be hardly adapted for distributed scatterers. However, in coastal zones over the sea, and in ocean scenes in highresolution images, many ambiguities due to distributed targets are present. For these situations, the use of techniques based on the concept of selective filtering has been advocated [5]-[7]. These techniques use a band-pass filter to select from the whole azimuth spectrum those areas that are less affected by ambiguities, so that this approach can be applied to ghosts due to both point targets and distributed targets, although at the cost of a resolution loss. More recently, a method exploiting the availability of multipolarization data has been also proposed [8]. However, we are here interested on methods able to deal with a single polarization channel, which have a wider range of applicability.

In this paper, we present a recently developed azimuth ambiguity filtering method [7] and we apply it as a preliminary step of an adaptive-threshold cell-averaging constant-false-alarm-rate (CFAR) ship detection algorithm [9]. We then show that use of this preliminary filtering step allows us to significantly improve the performance of the ship detection by reducing the false alarm rate, without reducing the detection rate. The approach is experimented and validated using a couple of Cosmo/SkyMed SAR images: one is relevant to the Gulf of Naples, Italy, and the other one to an area close to the coast of Malta.

\section{AZIMUTH AMBIGUITY FILTERING}

Recently, some of the authors of this paper have developed a method for the filtering of azimuth ambiguities on stripmap SAR images, named "asymmetric mapping and selective filtering" (AM\&SF) method [7]. This method is based on the theory of selective filtering and on a two-step procedure.

In the first step, two asymmetric filters are used to suppress ambiguities due to each sidelobe of the antenna pattern, and the ratios between the original and filtered images are used to produce two binary maps of the ambiguity-affected areas (one for each sidelobe). The transfer function of the filters is obtained using the theory of Wiener filtering, as in [5]: substantially, in the frequency domain each filter selects the region of the signal spectrum 
less affected by aliasing, i.e., the region where the null of the considered folded AAP sidelobe is located (in fact, the azimuth spectrum of the scene reflectivity is weighted by the AAP of the sensor, so that where the folded AAP presents a null, the spectrum of the ambiguity will be null, whatever the original shape of reflectivity spectrum). The expression of the filters' transfer functions (TFs) is given by

$$
H_{ \pm}(\xi)=\frac{\sigma_{0}}{\sigma_{ \pm}}\left(\frac{\left|W_{ \pm}(\xi)\right|^{2}}{\left|W_{0}(\xi)\right|^{2}}+\frac{\sigma_{t}}{\sigma_{ \pm}} \frac{1}{\left|W_{0}(\xi)\right|^{2}}+\frac{\sigma_{0}}{\sigma_{ \pm}}\right)^{-1}
$$

where $\xi$ is the azimuth wavenumber, related to the AAP angle $\psi$ by the relation $\xi \simeq-\frac{4 \pi}{\lambda} \psi ; \sigma_{0}, \sigma_{+}$, and $\sigma_{-}$are the power spectral densities of the reflectivity relevant to the unambiguous and to the two ambiguous targets, respectively; $\sigma_{t}$ is the white thermal noise power density; and $\left|W_{0}(\xi)\right|,\left|W_{+}(\xi)\right|$, and $\left|W_{-}(\xi)\right|$ represent the main beam and the two first sidelobes of the AAP, respectively. The filters of (1) are computed assuming that the ambiguity due to only one sidelobe at a time is present within an image pixel. In particular, in (1) the filter $H_{+}$is obtained assuming that $\sigma_{+} \gg \sigma_{0}$ and $\sigma_{-}=0$, while, conversely, for $H_{-} \sigma_{-} \gg$ $\sigma_{0}$ and $\sigma_{+}=0$. Instead, in [5] the filter transfer function is evaluated assuming that both ambiguities are very large, i.e. $\sigma_{+}=\sigma_{-}=\sigma \gg \sigma_{0}$, thus leading to the following expression:

$$
H(\xi)=\frac{\sigma_{0}}{\sigma}\left(\frac{\left|W_{+}(\xi)\right|^{2}+\left|W_{-}(\xi)\right|^{2}}{\left|W_{0}(\xi)\right|^{2}}+\frac{\sigma_{t}}{\sigma} \frac{1}{\left|W_{0}(\xi)\right|^{2}}+\frac{\sigma_{0}}{\sigma}\right)^{-1} .
$$

Use of two different filters and, hence, two different maps (one for each first sidelobe) for ghost detection, differently from [5], allows identification of ghosts even in cases in which the AAP and PRF value are such that the peak of one folded AAP sidelobe locates almost exactly in correspondence of the null of the other one. This is sometime the case for current high-resolution spaceborne SAR sensors, e.g. TerraSAR-X and Cosmo/SkyMed, and in such case the method in [5] fails to identify ghosts, whereas the AM\&SF method of [7] can identify them. In these cases, the filter in (2) is not adequately selective in the frequency domain. As an example in Fig. 1 (a) the first sidelobes of a typical TerraSAR-X AAP folded into the azimuth processed bandwidth are shown, while in Fig. 1 (b) the filter TF resulting from the definition in (2) is presented. It can be noted that the filter TF obtained through (2) is almost uniform in the processed bandwidth. Conversely, in Fig. 1 (c) and (d) the filter TFs of (1), $H_{+}$and $H_{-}$respectively, are shown: it is possible to appreciate the huge increase in frequency selectivity with respect to Fig. 1 (b).

In the second step, the maps of the ambiguity-affected areas are used to produce a final image in which only the areas affected by the ambiguities are replaced by their filtered versions, obtained via the proper of the two filters cited above.

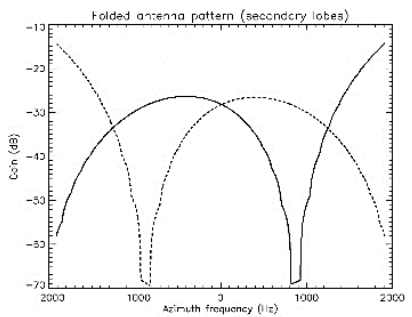

(a)

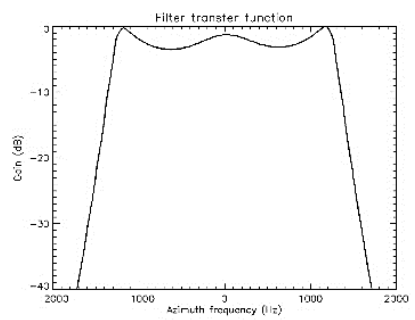

(c)

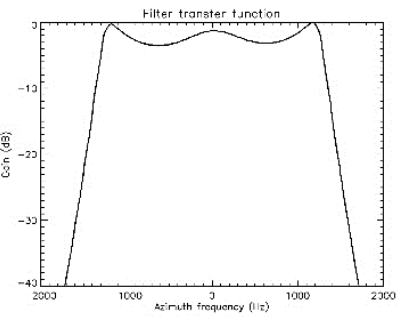

(b)

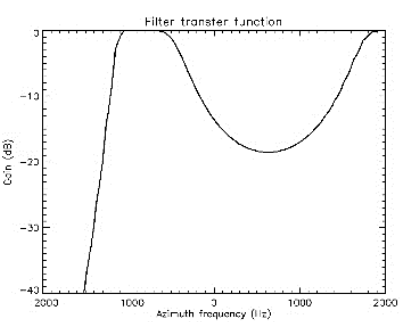

(d)
Fig. 1 First sidelobes of the AAP folded into the processed azimuth bandwidth (a) and filter TFs $H(\mathrm{~b}), H_{+}$(c), and $H_{-}$(d) in the case of TerraSAR-X data.

The entire procedure is very fast: processing time is 20 seconds for an about $12000 \times 9000$ pixels input SLC SAR image, on a $3 \mathrm{GHz}$ dual core PC, with $8 \mathrm{~GB}$ RAM, in normal working conditions, using non-optimized IDL ${ }^{\mathrm{TM}}$ software code. Note that processing time scales linearly with the total number of pixels. Examples of application of the azimuth filtering method are shown in Figs. 2-4. For a full description of the AM\&SF method, the reader is referred to [7].

\section{SHIP DETECTION}

The above described azimuth ambiguity filtering method has been applied as a preliminary step of a standard ship detection algorithm based on an adaptive-threshold cellaveraging CFAR method [9]. This algorithm, for each pixel of a multilook SAR image, computes the ratio between the mean intensity of a small box centered on the considered pixel (target box) and the mean intensity of a large area (background area), and identifies the pixel as belonging to a ship if this ratio is larger than a locally-computed threshold. The threshold is computed by prescribing a desired FAR and assuming a Gamma distribution for the background area. Computation of the threshold is similar to the one employed in the ratio-of-average (ROA) method of [10].

SAR ship detection takes a great advantage from azimuth ambiguity filtering. In particular, since ghosts are particularly contrasted in low backscattering sea areas close to the coast, due to azimuth ambiguity of coastal buildings and harbor infrastructures, CFAR detection may produce several false alarms on such sea areas. For this reason, CFAR is often optimized in order to decrease the number of false alarms in coastal areas, but this leads to a general 
reduction of the probability of detection also on offshore sea areas. Therefore, the suppression of ghosts allows a better exploitation CFAR techniques and the achievement of an overall improved detection capability (better Receiver Operating Characteristic (ROC) curve).

It is worth mentioning that CFAR techniques are based on simple statistical criteria to measure quantitatively the contrast of a target with respect to the background and to perform detection. For this reason, they offer an effective approach to measure the capability of the developed filter to suppress azimuth ambiguities.

In order to verify the improvement obtained by using the preliminary ghost filtering step, we have compared the performance of the ship detection algorithm applied to nonfiltered and filtered images. The considered images are two stripmap Cosmo/SkyMed images: one is relevant to the Gulf of Naples and the other one to an area close to the coast of Malta.

The first image is shown in Fig. 2 (a): azimuth ambiguities due to buildings and man-made areas are visible all over the sea surface. In this case, while on the nonfiltered image of Fig. 2 (a) a large number of ships is detected, corresponding to both actual ships and ghosts, on the filtered image of Fig. 1 (b) only actual ships are detected. Results of the ship detection in an inset of the nonfiltered and filtered images are depicted in Fig. 3 (a) and 3 (b), respectively.

Similar results are obtained for the second image, as illustrated in Fig. 4. In this case, the ambiguities are due to the ship present at the center of the subset reported in Fig. 4. In Fig. 4 (a) the two ghosts due to the first two sidelobes of the AAP are clearly visible on each side of the ship. The ghosts are wrongly detected as ships when the shipdetection algorithm is applied on the image in Fig. 4 (a). Also in this case applying the ship detection on the filtered image, we are able to remove the false alarms, while preserving the detection of the true target, as shown in Fig. 4 (b).

\section{CONCLUSIONS}

In this paper, the problem of false alarms due to SAR azimuth ambiguities in ship-detection applications has been discussed and a method for the reduction of these false alarms has been presented. In particular, the proposed method is based on the preliminary application of the $\mathrm{AM} \& \mathrm{SF}$ technique for filtering the ambiguities present on the sea surface and on the subsequent use of an adaptivethreshold cell-averaging CFAR ship-detection algorithm. The proposed approach has been tested on a couple of stripmap Cosmo/SkyMed images of maritime scenes. The obtained results demonstrate the possibility of drastically reducing the false-alarm rate, without decreasing the detection rate.

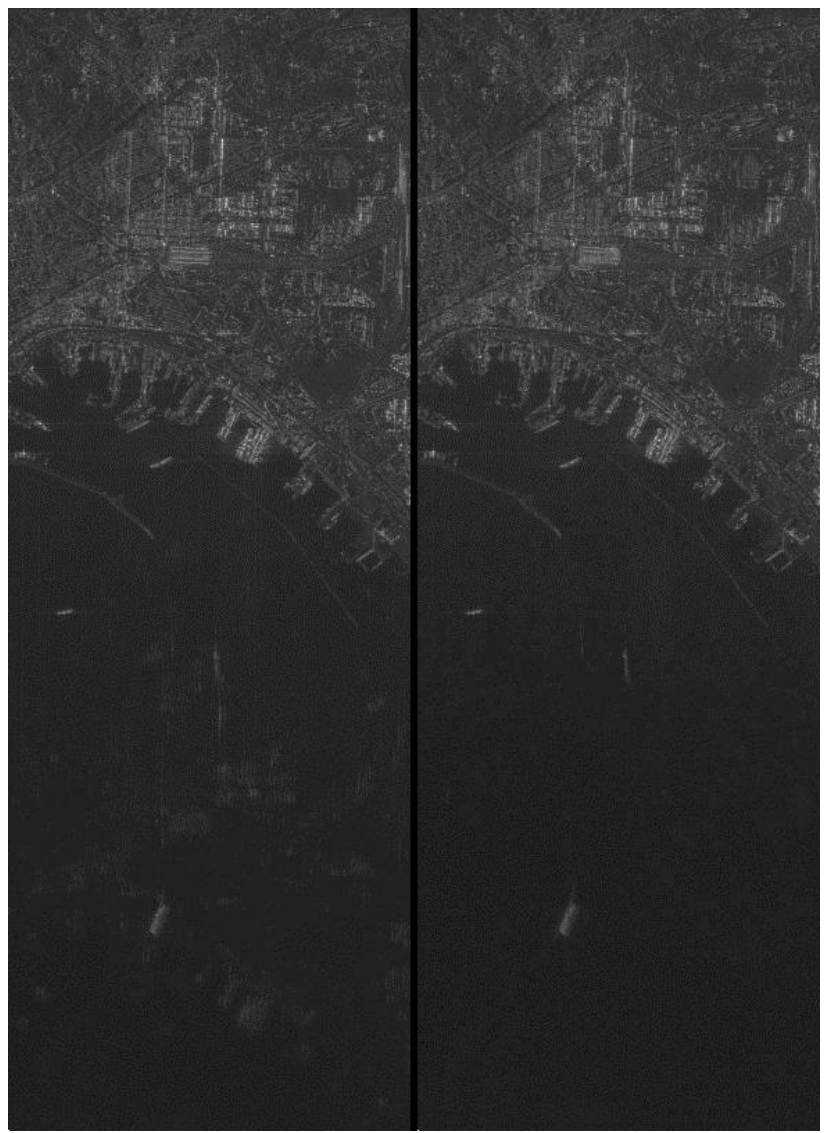

(a)

(b)

Fig. 2 Original Cosmo/SkyMed image of the gulf of Naples (a) and its filtered version (b) (a spatial multilook of 6x6 pixels is applied). Ambiguities due to buildings and man-made areas are visible all over the sea surface in (a), while they are strongly attenuated in (b), where only actual ships are visible on the sea.

\section{ACKNOWLEDGMENT}

This work was supported by ASI in the framework of the AO project "Buildings feature extraction from single SAR images: application to Cosmo/SkyMed high resolution SAR images" and by the EU-FP7 project "Development of PreOperational Services for Highly Innovative Maritime Surveillance Capabilities" (DOLPHIN).

\section{REFERENCES}

[1] S. Brusch, S. Lehner, T. Fritz, M. Soccorsi, A. Soloviev, and B. van Schie, "Ship Surveillance with TerraSAR-X," IEEE Trans. Geosci. Remote Sens., vol. 49, no. 3, pp. 1092-1103, Mar. 2011.

[2] A. Moreira, "Suppressing the azimuth ambiguities in synthetic aperture radar images," IEEE Trans. Geosci. Remote Sens., vol. 31, no. 4, pp. 885-895, July 1993.

[3] R. K. Raney and G. J. Princz, "Reconsideration of Azimuth Ambiguities in SAR," IEEE Trans. Geosci. Remote Sens., vol. 25, no. 6, pp. 783-787, Nov. 1987. 


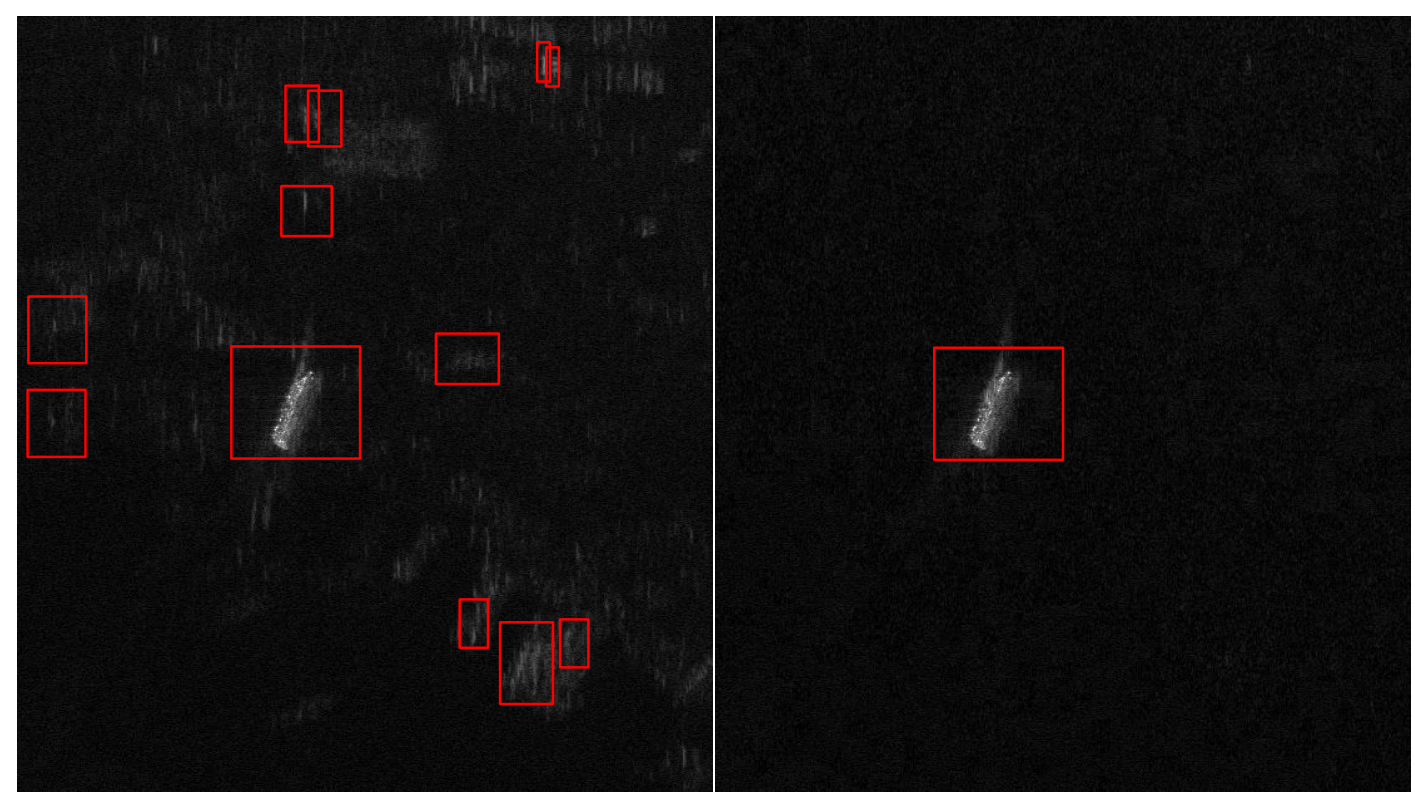

(a)

(b)

Fig. 3 Full-resolution particular of the Cosmo/SkyMed image in Fig. 1: original (a) and filtered (b) image. Targets identified as ships by the ship-detection algorithm are enclosed in the red boxes. It is evident that no false alarm is obtained by using the preliminary azimuth ambiguity filtering step.

[4] J. Chen, M. Iqbal, W. Yang, P. Wang, and B. Sun, "Mitigation of Azimuth Ambiguities in Spaceborne Stripmap SAR Images Using Selective Restoration," IEEE Trans. Geosci. Remote Sens., vol. 52, no. 7, pp. 4038-4045, July 2014.

[5] A. Monti Guarnieri, "Adaptive Removal of Azimuth Ambiguities in SAR Images," IEEE Trans. Geosci. Remote Sens., vol. 43, no. 3, pp. 625-633, Mar. 2005.

[6] F. K. Li and W. T. K. Johnson, "Ambiguities in Spaceborne Synthetic Aperture Radar Systems," IEEE Trans. Aerosp. Electron. Syst., vol. 19, no. 3, pp. 389-397, May 1983.

[7] G. Di Martino, A. Iodice, D. Riccio, and G. Ruello, "Filtering of Azimuth Ambiguity in Stripmap Synthetic Aperture Radar Images," IEEE J. Sel. Top. Appl. Earth Obs. Remote Sens., 2014, in print.

[8] D. Velotto, M. Soccorsi, and S. Lehner, “Azimuth Ambiguities Removal for Ship Detection Using Full Polarimetric X-Band SAR Data," IEEE Trans. Geosci. Remote Sens., vol. 52, no. 1, pp. 7688, Jan. 2014.

[9] D. J. Crisp, "The state-of-the-art in ship detection in synthetic aperture radar imagery," Intell., Surveillance and Reconnaissance Div., Inf. Sci. Lab., Def., Sci. Technol. Org., Edinburgh, S.A., Australia, May 2004, Res. Rep. DSTO-RR-0272.

[10] R. Touzi, A. Lopes, and P. Bousquet. "A statistical and geometrical edge detector for SAR images." IEEE Trans. Geosci. Remote Sens. vol. 26, no. 6, pp. 764-773, 1988.

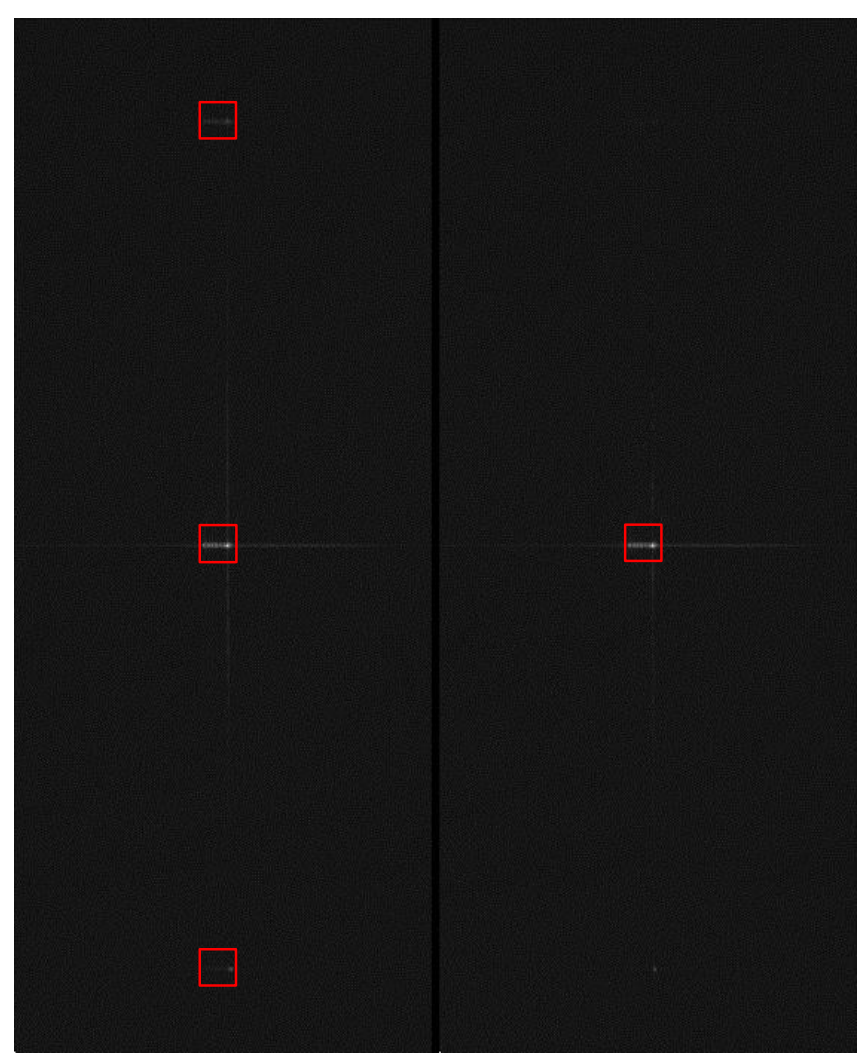

(a)

(b)

Fig. 4 Original Cosmo/SkyMed image acquired close to the coast of Malta (a) and its filtered version (b) (a spatial multilook of 8x8 pixels is applied). Targets identified as ships by the ship-detection algorithm are enclosed in the red boxes. 